\title{
Biomarkers of intestinal epithelial damage in children with food allergies
}

\author{
Artur Rycyk, Dariusz M. Lebensztejn, Beata Cudowska \\ Department of Pediatrics, Gastroenterology, Hepatology, Nutrition and Allergology, Medical University of Bialystok, \\ Bialystok, Poland
}

\section{ABSTRACT}

Food allergy has a significant negative impact on patients and their families, and it has become an important public health problem. Currently used diagnostic methods, such as skin prick tests (SPT) and determination of specific immunoglobulins E (sIgE), present limited sensitivity and specificity. Therefore, new tools are expected to improve the accuracy of the diagnostic work-up. A recent hypothesis of allergic reactions indicates a primary role of dysregulation of the epithelial barrier, which pioneers the role of biomarkers of intestinal damage in food allergy. The objective of this paper is to present the currently most promising biomarkers for the diagnosis, prognosis, and management of food allergy in children.

\section{KEY WORDS:}

children, food allergy, biomarkers.

\section{INTRODUCTION}

The diagnosis of food allergy (FA) in children is difficult because until now there has been no single, universal diagnostic method. The diagnostic difficulties result from the variety of offending foods, the variability of their sensitising properties, and the various mechanisms of allergic reactions. For these reasons, clinicians are still looking for more accurate diagnostic tools.

A recent hypothesis on the pathogenesis of allergic reactions indicates a crucial role of epithelial barrier impairment. According to Zissler et al., the exploration of biomarkers for allergic rhinitis and allergic asthma has revealed 161 different, potential biomarkers; about 20 of them are exclusively derived from epithelium of the respiratory tract [1]. In terms of gastrointestinal (GI) diseases, until now biomarkers of the epithelial damage have been included in practice guidelines in the management of inflammatory bowel disease (IBD). Furthermore, there is the potential for more epithelial-derived markers to be incorporated into diagnostic standards of such clinical entities as IBD, irritable bowel syndrome (IBS), short bowel syndrome, colorectal cancer, malabsorption syndrome, or FA [2].

This paper summarises the current state of knowledge on selected, most promising biomarkers of intestinal epithelial damage that might be useful in FA diagnostics.

\section{GASTROINTESTINAL FOOD ALLERGY}

FA can be defined as an abnormal immune response on exposure to consumed food, appearing reproducibly, and causing adverse reactions $[3,4]$.

According to current nomenclature, immunological mechanisms involved in allergy are classified as either immunoglobulin E (IgE)-mediated, non-IgE-mediated, or a mixture of IgE- and non-IgE-mediated [3]. In the ma-

\section{ADDRESS FOR CORRESPONDENCE:}

Artur Rycyk, Department of Paediatrics, Gastroenterology, Hepatology, Nutrition and Allergology, Medical

University of Bialystok, 17 Waszyngtona St., 15-274 Bialystok, Poland, ORCID: 0000-0002-8132-1345,

e-mail: artur.rycyk@gmail.com 
jority of cases, allergic diseases with GI manifestation are considered to be non-IgE-mediated; however, they might be presented with different immunological mechanisms as well [3].

The highest prevalence of FA is reported among the youngest children (up to $6-8 \%$ of the infant population), decreasing over the years, to around $3-4 \%$ of adolescents and $1-3 \%$ of adults [4].

GI allergies are classified by differences in symptomatology into various clinical entities. Food protein-induced enterocolitis syndrome (FPIES) is a severe non-immunoglobulin, IgE-mediated, gastrointestinal food hypersensitivity, which in an acute form manifests as intensive, repetitive vomiting, often accompanied with diarrhoea, leading to dehydration and lethargy. Together with food-protein-induced enteropathy (FPIE) and food protein-induced allergic proctocolitis (FPIAP) FPIES belongs to a group of non-IgE-mediated gastrointestinal food allergic disorders (non-IgE-GI-FA). These diseases primarily affect infants and are most commonly caused by cow's milk, soy, and rice proteins, although other food allergens can be triggers as well [5].

The diagnosis is based upon the presence of consistent clinical features with improvement following withdrawal of the suspected causal allergens. The results of skin prick tests or allergen-specific IgE antibodies are usually negative. In endoscopy, non-characteristic changes are manifested in the form of diffuse and/or haemorrhagic inflammation, without or with the involvement of the ileum and atrophy of the intestinal villi. Due to the lack of specific symptoms and laboratory tests, diagnosis of non-IgEGI-FA is often challenging and time-consuming $[4,5]$.

\section{THE FUNCTION OF THE INTESTINAL EPITHELIAL BARRIER}

The epithelial layer is a structure forming a barrier that separates the human host from the external environment. Together with gut-associated lymphatic tissue, it is the part of the largest immunological organ of the human body [6].

The intestinal barrier is established by a single layer of epithelial cells organised into crypts and villi. It is continuously renewed by multipotential intestinal epithelial stem cells. The integrity of the epithelial barrier rests upon the structure of cells and their junctional complexes: desmosomes, zonula adherens, and tight junctions. In response to a variety of stimuli, tight junctions are continuously opened and closed creating the dynamic functional state of the intestinal barrier.

More and more experimental and clinical evidence suggests that the two major factors capable of altering intestinal permeability are high-energy diet and gut microbiota modifications [7].

Depending on the dose and strain, microbes of the human intestine are able to influence, via various mechanisms, the barrier strength and its functional integrity.
First of all, some probiotics, like Lactobacillus plantarum MB452, have been proven to directly alter tight junction protein expression [8]. Moreover, bacterial influence on gut permeability also results from releasing a variety of molecules: soluble peptides, cellular structural components, metabolites, or even toxins [9]. Those bacterial metabolites, called postbiotics, including short-chain fatty acids - acetate and butyrate, have been shown to directly enhance the intestinal epithelial barrier function and therefore protect against pathogens $[10,11]$. What is more, microbes can influence the epithelial barrier also in an indirect manner - through their impact on host immune cells and the release of cytokines [12].

Manipulation of the gut microflora with probiotics, antibiotics, or microbial products results in both an attenuation of disease and a restoration of normal gut permeability [9].

According to pathophysiology of allergy, dysregulation of a T-helper type- 2 response and hypersensitivity reactions upon antigen re-exposure are the key mechanisms that initiate IgE-sensitisation and recruitment of mast cells in the gastrointestinal mucosa. These pre-sensitised mast cells can be triggered directly by allergens in the lamina propria. During an effector phase of allergic inflammation, recruitment of mononuclear inflammatory cells results in the release of a number of proinflammatory cytokines as well as mast cell degranulation, which leads to increased intestinal permeability and transepithelial allergen transport, thereby inducing the inflammatory reaction [13].

\section{BIOMARKERS OF GASTROINTESTINAL INFLAMMATION}

The World Health Organisation (WHO) defines a "biological marker" as "any substance, structure, or process that can be measured in the body or its products whose detection indicates a particular disease state" [14]. The term "biomarker" contains multiple categories of disease indicators, including disease-specific molecules such as pathogen-specific proteins, host-response molecules such as immunoglobulins, physiological measurements such as blood pressure, or results from imaging technologies.

A useful biomarker should directly and uniquely identify a disease-causing agent, be easy to detect in multiple settings, and clearly identify whether a disease is currently active [15]. Nowadays, searching for proper biomarkers is one of the main research targets throughout medicine.

\section{SUGAR ABSORPTION TEST}

The non-invasive assessment of intestinal permeability in humans has been known for almost 30 years and served mainly as diagnostic screening for small bowel diseases. The basic tool for epithelial permeability assessment is the sugar absorption test (SAT). It is based on passive absorption of probes from the GI tract and com- 
plete elimination by the urinary tract. After 5-6 hours of urine collection, samples are analysed using high-pressure liquid chromatography (HPLC) or liquid chromatography in combination with mass spectrometry (LC/MS).

The permeability of selected parts of the intestine is measured by applying specific probes: for assessment of stomach and proximal duodenum permeability - sucrose; for small intestine - lactulose; and for the whole gut - sucralose [16]. Using their combination, isolated colon permeability can be evaluated by 24-hour lactulose excretion subtracted from 24-hour sucrose excretion [17]. For this reason, a recent approach for assessing gut permeability is to use "multisugar tests", like the one based on a combination of sucrose, lactulose, sucralose, erythritol, and rhamnose simultaneously [18].

However, until now, the lactulose-to-mannitol ratio (LMR) has been the most widely used measurement for routine diagnostics and scientific purposes [19]. Lactulose is a large molecule that consists of galactose and fructose. Due to the lack of specific enzymes converting lactulose in human intestine, this disaccharide is the most reliable measure for diagnostic purposes. Quantities of lactulose that are absorbed through intercellular spaces indicate small intestinal damage. In further parts of the GI tract lactulose is metabolised by residual bacteria or neutralised by other agents like mannitol. In contrast, mannitol is a small monosaccharide that is absorbed via trans/paracellular pathways of healthy small intestine and absorption of which is strictly dependent on the surface of the villi area. Determination of the probe ratio, rather than the rate of movement of any one probe, minimises confounding factors such as defects in collection, gastric retention, transit time, and renal clearance. LMR advantages include the following: safety, low cost, and satisfactory correlation with intestinal pathophysiology. As well as it being time consuming, Denno et al. revealed other limitations LMR such as: lack of standardisation of assay method and specimen collection, and no standard values for specific populations [20].

Increased permeability detected by LMR has served as a screening method for enteropathy and FA as well as response to treatment, and for assessing the prognosis in coeliac disease and Crohn's disease [21]. The role of increased intestinal permeability in developing FA was confirmed in a group of patients on a calcineurin inhibitor therapy after solid organ transplantation [22]. Kalach et al. concluded that LMR with a cut-off level of $3.95 \%$ reached a performance value of $76 \%$, which exceeded the levels of IgE, IgG, skin prick test, or atopy patch test [23]. The authors underlined that the highest sensitivity was observed in patients with non-IgE-mediated allergy.

\section{CITRULLINE}

A growing number of data support the implementation of citrulline as a non-invasive, simple, and accurate tool for estimating intestinal permeability.
Citrulline is an amino acid produced from glutamine by intact small bowel enterocytes. Recent studies revealed that citrulline plasma concentration presents direct correlation with the mass of intestinal epithelial cells and thereby can be used as a marker of gut status in short bowel syndrome [24].

A study conducted on patients with coeliac and non-coeliac villous atrophy revealed that citrulline levels were significantly lower than in controls and increased during a year of successful treatment [25]. Other possible clinical indications for citrulline assessment include detection of intestinal damage during chemotherapy and necrotising enterocolitis (NEC) [26, 27].

In comparison to LMR, citrulline has been proven to be a more sensitive and specific method for bowel leakage diagnostics [28]. In a recently published systematic review and meta-analysis, it was estimated that its sensitivity and specificity on the level $\sim 80 \%$ indicates intestinal insufficiency [29]. In a study of non-invasive biomarkers for assessment of villous abnormalities citrulline was proven to be the most reliable [30].

In terms of allergic diagnostics, citrulline may replace LMR in assessing intestinal permeability and seems to be a particularly promising marker for food-induced enteropathy. However, until now no sufficient data has been collected to establish citrulline's role in FA diagnostics.

\section{INTESTINAL-FATTY ACID BINDING PROTEIN}

Another diagnostic tool for assessing epithelial cell integrity is intestinal-fatty acid binding protein (I-FABP). It is a member of larger protein family of fatty acid binding proteins (FABPs) that until now have been identified in such organs as the heart, intestine, liver, epidermal layer of skin, muscle, and fatty tissue. FABPs are small, water-soluble molecules that transport fatty acids from apical membrane of enterocyte to the endoplasmatic reticulum for further biochemical reactions. Depending on the localisation in the GI system, three types of FABP were identified: intestinal FABP (I-FABP), found predominantly in the jejunum; liver FABP (L-FABP), derived mostly from liver; and ileal bile acid binding protein (I-BABP), exclusively bound to the ileum. The concentration of all FABPs can be obtained from plasma, serum, or urine using an enzyme-linked immunosorbent assay (ELISA) [31].

Urine measurements present a valuable alternative for circulating I-FABS with the advantage of relatively non-invasive (and blood sparing) sample collection and detection over a longer period of time (half the time of circulating I-FABP - 11 minutes) [7].

I-FABP basal levels reflect the physiological turnover rate of enterocytes, which was confirmed by Derikx et al. [32]. This study involved a group of 34 patients with intestinal mucosal barrier injury resulting from myeloab- 
lative therapy, presenting significant decrease in serum levels of I-FABP and I-BABP from the day of transplantation until day 14 .

Elevated levels of both circulating and urine I-FABP are believed to indicate intestinal epithelial cell damage, which has been observed in patients with intestinal ischaemia, systemic inflammatory response syndrome (SIRS), and NEC [31,33]. Schurink et al. showed that in terms of NEC, urine I-FABP could serve as a reliable biomarker for complicated course of disease; even in the earliest stages of the disease [7]. On the other hand, serum concentration of I-FABP has also been applied for the assessment of disease activity in celiac disease [34]. Therefore, I-FABP has emerged as a possible non-invasive marker for evaluating gut wall integrity loss and inflammation in FA. However, further studies are required to confirm such predictions.

\section{REGENERATING GENE $1 \alpha$}

Studies on the pathophysiology of autoimmune diseases led to the discovery of the regenerating protein (Reg) family. They are involved in the process of cell proliferation, differentiation, inflammation, and carcinogenesis [35]. In humans, until now, five functional Reg genes have been isolated: REG1 $\alpha, R E G 1 \beta, R E G 3, H I P / P A P$, and $R E G 4$ [36]. Reg family proteins were identified as acute phase reactants, anti-apoptotic factors, and growth agents [36].

Primarily, regenerating gene $1 \alpha(R E G 1 \alpha)$ expression was found in various types of tissues in the course of autoimmune diseases, like in salivary glands in Sjögren's syndrome or in pancreatic islets in type 1 diabetes. Further studies revealed the highest expression of REG1a within the small intestine [35]. Until now REG1a involvement was confirmed in such diseases as gastritis, pancreatitis, GI cancer, IBD, and cystic fibrosis [35]. However, at this point the most promising field for REG1 $\alpha$ practise application is celiac disease. In a preliminary study by Planas et al. increased serum levels of REG1 a reflected the destructive process of the villi, followed by significant reduction after a gluten-free diet [37]. Singh et al. compared serum concentration of REG1 $\alpha$ and plasma levels of I-FABP with their tissue expression in duodenal biopsies in 131 patients with CD, before and after a gluten-free diet. The results showed that levels of both, I-FABP and REG1 $\alpha$ were significantly higher before treatment, while their tissue expression was lower before treatment. After introduction of a gluten-free diet, a decrease in plasma I-FABP and serum REG1a levels was observed, parallel to a significant increase of tissue expression [30].

Because celiac disease and FA share a similar pathophysiological process of immunological inflammation, biomarkers like REG1a might have diagnostic value for both of them.

\section{FAECAL CALPROTECTIN}

The allergic inflammation process developing within intestinal wall involves multicellular infiltration including neutrophils. Activated neutrophils infiltrate the gut mucosa and products of their activation, like faecal calprotectin (FC), can be later detected in faeces [38].

FC is a zinc- and calcium-binding protein complex composed of one light and two heavy chains of the S100 family. It is a main neutrophil cytosol protein of a chemotactic, antibacterial, and proliferative properties. Although mostly associated with neutrophils, in smaller amounts, calprotectin is also present in monocytes, macrophages, bone marrow cells, squamous epithelial cells, mucosal epithelial cells, microvascular endothelial cells, and activated fibroblasts [39]. FC is known to be resistant to proteolytic enzymes, which makes it an easily accessible and reliable diagnostic tool [38].

Increased levels of FC indicate and closely correlate with the severity of inflammatory infiltration within the intestinal wall [40]. Furthermore, elevated FC may even precede typical inflammatory findings in endoscopic examination, which has been observed in IBD [41]. However, it should be remembered that the concentration of FC in stool varies with age. The cut-off point for adults and children over four years of age was established at the level of $50 \mu \mathrm{g} / \mathrm{g}$ [42]. In terms of IBD diagnostics, higher sensitivity was observed for concentrations over $100 \mu \mathrm{g} / \mathrm{g}$ [43]. Cut-off points in younger age groups are more difficult to present because the level of FC depends on a number of factors, such as gestational and/or postnatal age or feeding patterns with either breastmilk or milk formula. In a study by Ezri et al. the cut-off level was found to be $<350 \mu \mathrm{g} / \mathrm{g}$ in the first year of life and $<275 \mu \mathrm{g} / \mathrm{g}$ during childhood [44].

Based on literature overview, FC measurement in children was valuable in the diagnostics of IBD, infectious diarrhoea, GI complications in juvenile idiopathic arthritis, NEC, colonic polyps, non-steroidal anti-inflammatory drug-induced enteropathy, cystic fibrosis, and follow-up in coeliac disease $[45,46]$. Until now its most significant role is focused on diagnosis, the monitoring of remission and mucosal healing, and in the prediction of relapse in paediatric IBD.

Nevertheless, a growing body of clinical data suggest that FC is a valuable test for FA diagnostics in children [23, 45-48]. Waligora-Dupriet et al. found that the level of FC in infants with FA was twice that of those without FA [45]. Beser et al. indicated that in non-IgE-mediated phenotypes of FA, FC tends to present significantly higher levels than in IgE-mediated ones $(889 \pm 278 \mu \mathrm{g} / \mathrm{g}$ vs. 392 $\pm 209 \mu \mathrm{g} / \mathrm{g}$ ), leading to the conclusion that it is also more useful to determine relapses and the follow-up of patients in the non-IgE-mediated group [47]. Finally, Kukkonen et al., in a prospective study upon over 230 children participating in a randomised double-blind placebo- 
controlled allergy-prevention trial with probiotic strains, noted that increased calprotectin levels at six months of age were associated with lower risk of later atopic sensitisation [48].

\section{EOSINOPHIL-DERIVED NEUROTOXIN}

As mentioned above, allergic inflammation is associated with recruitment of leukocytes, particularly eosinophils. They are mobilised in response to Th2 stimuli characteristic for allergic inflammation and parasitic helminth infection. Parallel to neutrophils, their activation leads to the release four major cytosolic proteins, including eosinophil-derived neurotoxin (EDN) [49]. From previous studies, it is also known that eosinophil protein $\mathrm{X}$ (the terms EPX and EDN refer to the same molecule) together with eosinophil cationic protein (ECP) belong to the so-called RNase A superfamily [49]. Since its first purification in 1981, scientists have uncovered neurotoxic, chemotactic, transductive (via Toll-like receptors), and antiviral properties - particularly against respiratory syncytial virus (RSV) [50].

EDN concentration was studied in plasma, urine, and stools in a number of inflammatory diseases, including asthma and atopic dermatitis [51-53]. In terms of FA, Ada et al. studied a group of children diagnosed with FPIES, in whom the level of EDN in stools was tested before and after oral food challenge. It revealed strong positive correlation of the biomarker, reaching its maximum concentration after 24 hours of exposure [53]. Further studies confirmed the findings for FPIP and FPIE, together with an observation of particularly high EDN levels in bloody stools [54]. Kalach et al. estimated the diagnostic accuracy for EDN to be $72 \%$ and concluded that faecal EDN appeared to be more sensitive and closely correlated with the severity of illness than blood and urinary measurements in atopic dermatitis and atopic asthma [23].

\section{TUMOUR NECROSIS FACTOR $\alpha$}

The last but not least promising biomarker of FA is tumour necrosis factor $\alpha$ (TNF- $\alpha)$. Since the discovery of
TNF in 1962, research has revealed a further 19 agents, creating the TNF superfamily and demonstrating their proinflammatory properties. The role of TNF- $\alpha$ in inflammation, apoptosis, proliferation, angiogenesis, metastasis, and morphogenesis has been well-documented and used in diagnostics in neurology (depression, bipolar disorder, epilepsy, Alzheimer's disease, Parkinson's disease, multiple sclerosis), pulmonary diseases (asthma, chronic bronchitis, chronic obstructive pulmonary disease, acute lung injury, acute respiratory distress syndrome), and autoimmune diseases (uveitis, multiple sclerosis, systemic lupus, arthritis, psoriasis, and Crohn's disease) [55].

Currently it is believed that TNF- $\alpha$ also plays a crucial part in the pathophysiology of allergy. Two separate studies by Hayman et al. and Rodriguez et al. have shown that TNF- $\alpha$ initiates a process of increased intestinal permeability $[56,57]$. This role of TNF- $\alpha$ was confirmed by further studies indicating high molecule expression in epithelial cells and mononuclear blood cells derived from the intestines of patients with FPIES [58]. It is interesting that TNF- $\alpha$ was also detected in the stools of patients undergoing oral food challenge with confirmed cow's milk protein allergy [59]. Upon antigen-specific cytokine secretion profiles of 89 blood samples from Japanese children with FA Morita et al. concluded that particularly high levels of TNF- $\alpha$ indicate a critical role of that cytokine in intestinal epithelial cell damage and eosinophil infiltration [60]. The authors suggest that utility of TNF- $\alpha$ testing expands beyond FPIES and can be useful for both IgE-mediated and non-IgE-mediated subtypes of allergy. Further studies are warranted to confirm the diagnostic value of TNF- $\alpha$.

\section{CONCLUSIONS}

The presented paper indicates that there are a number of promising markers for non-IgE-mediated FA diagnostics (Table 1). Some of them, such as LMR and FC, seem to play a well-established role in the process of diagnosing and monitoring the disease's activity. Others, like citrulline, EDN, and TNF- $\alpha$, need further prospective confirmatory studies on a larger scale. However, it is unlikely that any of the selected methods will become a single,

TABLE 1. Potential biomarkers according to their origin (epithelium, infiltrate derived and matrix)

\begin{tabular}{|c|c|c|c|c|c|c|c|c|}
\hline \multicolumn{2}{|l|}{ Origin } & LMR & Citrulline & $\mathrm{FC}$ & I-FABP & REG1a & EDN & TNF- $a$ \\
\hline \multirow{3}{*}{$\begin{array}{l}\text { Epithe- } \\
\text { lium } \\
\text { derived }\end{array}$} & Enterocyte integrity & $x$ & $x$ & & & & & \\
\hline & Enterocyte injury & & & $X$ & $x$ & & & \\
\hline & Enterocyte regeneration & & & & & $x$ & & \\
\hline \multicolumn{2}{|c|}{ Infiltrate derived } & & & & & & $x$ & $x$ \\
\hline \multirow[t]{4}{*}{ Matrix } & Blood & $x$ & $x$ & & $x$ & $x$ & $x$ & $x$ \\
\hline & Urine & $x$ & & & $x$ & & $x$ & \\
\hline & Faeces & & & $x$ & & & & $x$ \\
\hline & Biopsies & & $x$ & & $x$ & $x$ & & \\
\hline
\end{tabular}

LMR-lactulose-to-mannitol ratio, FC - faecal calprotectin, I-FABP - intestinal fatty acid binding protein, REG1a - regenerating gene 1a, EDN - eosinophil-derived neurotoxin, TNF- $a$ - tumour necrosis factor a 
stand-alone test, particularly in terms of the delayed onset, non-IgE-mediated mechanism, which is most common for FA. It is more probable that the combination of those will meet satisfactory levels of sensitivity and specificity for simple, accurate, and non-invasive FA diagnostics.

\section{DISCLOSURE}

The authors declare no conflict of interest.

\section{REFERENCES}

1. Zissler UM, Esser-von Bieren J, Jakwerth CA, et al. Current and future biomarkers in allergic asthma. Allergy 2016; 71: 475-494.

2. Siddiqui I, Majid H, Abid S. Update on clinical and research application of fecal biomarkers for gastrointestinal diseases. World J Gastrointest Pharmacol Ther 2017; 8: 39-46.

3. Muraro A, Werfel T, Hoffmann-Sommergruber K, et al. EAACI food allergy and anaphylaxis guidelines: diagnosis and management of food allergy. Allergy 2014; 69: 1008-1025.

4. Kaczmarski M, Wasilewska J, Jarocka-Cyrta E, et al. Polskie stanowisko w sprawie alergii pokarmowej u dzieci i młodzieży. Post Dermatol Alergol 2011; 28: 75-115.

5. Cudowska B, Lebensztejn DM. Zapalenie jelit indukowane białkami pokarmowymi u dzieci. Gastroenterol Prakt 2017; 1: 43-45.

6. Chehade M, Mayer L. Oral tolerance and its relation to food hypersensitivities. J Allergy Clin Immunol 2005; 115: 3-12.

7. Schurink M, Kooi EM, Hulzebos CV, et al. Intestinal Fatty Acid-Binding Protein as a Diagnostic Marker for Complicated and Uncomplicated Necrotizing Enterocolitis: A Prospective Cohort Study. PLoS One 2015; 10: e0121336.

8. Anderson RC, Cookson AL, McNabb WC, et al. Lactobacillus plantarum MB452 enhances the function of the intestinal barrier by increasing the expression levels of genes involved in tight junction formation. BMC Microbiol 2010; 10: 316.

9. Ewaschuk J, Diaz H, Meddings L, et al. Secreted bioactive factors from Bifidobacterium infantis enhance epithelial cell barrier function. Am J Physiol 2008; 295: 1025-1034.

10. Fukuda S, Toh H, Hase K, et al. Bifidobacteria can protect from enteropathogenic infection through production of acetate. Nature 2011; 469: 543-547.

11. Hamer HM, Jonkers D, Venema K, et al. Review article: the role of butyrate on colonic function. Aliment Pharmacol Ther 2008; 27: 104-119.

12. Arrieta MC, Bistritz L, Meddings JB, et al. Alterations in intestinal permeability. Gut 2006; 55: 1512-1520.

13. Mattila $P$, Joenväärä $S$, Renkonen J, et al. Allergy as an epithelial barrier disease. Clin Transl Allergy 2011; 1: 5.

14. WHO International Programme on Chemical Safety Biomarkers in Risk Assessment: Validity and Validation 2001. http://www.who. int/iris/handle/10665/42363

15. Byrnes SA, Weigl BH. Selecting analytical biomarkers for diagnostic applications: a first principles approach. Expert Rev Mol Diagn 2018; 18: 19-26.

16. Bischoff SC, Giovanni B, Buurman W, et al. Intestinal permeability - a new target for disease prevention and therapy. BMC Gastroenterol 2014; 14: 189.

17. Anderson $\mathrm{AD}$, Jain $\mathrm{PK}$, Fleming $\mathrm{S}$, et al. Evaluation of a triple sugar test of colonic permeability in humans. Acta Physiol Scand 2004; 182: 171-177.
18. van Wijck K, Verlinden TJ, van Eijk HM, et al. Novel multi-sugar assay for site-specific gastrointestinal permeability analysis: a randomized controlled crossover trial. Clin Nutr 2013; 32: 245-251.

19. DeMeo MT, Mutlu EA, Keshavarzian A, et al. Intestinal permeation and gastrointestinal disease. J Clin Gastroenterol 2002; 34: 385-396.

20. Denno DM, VanBuskirk K, Nelson ZC, et al. Use of the Lactulose to Mannitol Ratio to Evaluate Childhood Environmental Enteric Dysfunction: A Systematic Review. Clin Infect Dis 2014; 59: 213-219.

21. Vilela EG, Torres HO, Ferrari ML, et al. Gut permeability to lactulose and mannitol differs in treated Crohn's disease and celiac disease patients and healthy subjects. Braz J Med Biol Res 2008; 41: 1105-1109.

22. Boyle RJ, Hardikar W, Tang ML. The development of food allergy after liver transplantation. Liver Transpl 2005; 11: 326-330.

23. Kalach N, Kapel N, Waligora-Dupriet AJ. Intestinal permeability and fecal eosinophil-derived neurotoxin are the best diagnosis tools for digestive non-IgE-mediated cow's milk allergy in toddlers. Clin Chem Lab Med 2013; 51: 351-361.

24. Diamanti A, Panetta F, Gandullia P, et al. Plasma citrulline as marker of bowel adaptation in children with short bowel syndrome. Langenbecks Arch Surg 2011; 396: 1041-1046.

25. Crenn P, Vahedi K, Lavergne-Slove A, et al. Plasma citrulline: A marker of enterocyte mass in villous atrophy-associated small bowel disease. Gastroenterology 2003; 124: 1210-1219.

26. Barzał JA, Szczylik C, Rzepecki P, et al. Plasma citrulline level as a bio-marker for cancer therapy-induced small bowel mucosal damage. Acta Biochim Pol 2014; 61: 615-631.

27. Ioannou HP, Diamanti E, Piretzi K, et al. Plasma citrulline levels in preterm neonates with necrotizing enterocolitis. Early Hum Dev 2012; 88: 563-566.

28. Lutgens LC, Blijlevens NM, Deutz NE, et al. Monitoring myeloablative therapy-induced small bowel toxicity by serum citrulline concentration: a comparison with sugar permeability tests. Cancer 2005; 103: 191-199.

29. Konstantinos CF, Alastair F. Citrulline as a marker of intestinal function and absorption in clinical settings: A systematic review and meta-analysis. United European Gastroenterol J 2018; 6: 181191.

30. Singh A, Verma A, Das P, et al. Non-Invasive Biomarkers for Assessment of Villous Abnormalities in Patients with Celiac Disease and Other Enteropathies: An Alternative to Mucosal Biopsies. Gastroenterology 2017; 152: 264.

31. Coufal S, Kokesova A, Tlaskalova-Hogenova H, et al. Urinary Intestinal Fatty Acid-Binding Protein Can Distinguish Necrotizing Enterocolitis from Sepsis in Early Stage of the Disease. J Immunol Res 2016; 2016: 5727312

32. Derikx JP, Blijlevens NM, Donnelly JP, et al. Loss of enterocyte mass is accompanied by diminished turnover of enterocytes after myeloablative therapy in haematopoietic stem-cell transplant recipients. Ann Oncol 2009; 20: 337-342.

33. Bischoff SC, Barbara G, Buurman W, et al. Intestinal permeability - a new target for disease prevention and therapy. BMC Gastroenterology 2014; 14: 189.

34. Adriaanse MP, Tack GJ, Passos VL, et al. Serum I-FABP as marker for enterocyte damage in coeliac disease and its relation to villous atrophy and circulating autoantibodies. Aliment Pharmacol Ther 2013; 37: 482-490.

35. Zhang YW, Ding LS, Lai MD. Reg gene family and human diseases. World J Gastroenterol 2003; 9: 2635-2641.

36. Yoshimoto K, Fujimoto T, Itaya-Hironaka A, et al. Involvement of autoimmunity to REG, a regeneration factor, in patients with primary Sjögren’s syndrome. Clin Exp Immunol 2013; 174: 1-9. 
37. Planas R, Pujol-Autonell I, Ruiz E, et al. Regenerating gene I $\alpha$ is a biomarker for diagnosis and monitoring of celiac disease: a preliminary study. Transl Res 2011; 158: 140-145.

38. Fagerhol MK. Calprotectin, a faecal marker of organic gastrointestinal abnormality. Lancet 2000; 356: 1783-1784.

39. Yui S, Nakatani Y, Mikami M. Calprotectin (S100A8/S100A9), an inflammatory protein complex from neutrophils with a broad apoptosis-inducing activity. Biol Pharm Bull 2003; 26: 753-760.

40. Bunn SK, Bisset WM, Main MJ, et al. Fecal Calprotectin: Validation as a noninvasive Measure of Bowel Inflammation in Childhood Inflammatory Bowel Disease. J Pediatr Gastroentreol Nutr 2001; 33: 14-22.

41. Gisbert JP, McNicholl AG. Questions and answers on the role of faecal calprotectin as a biological marker in inflammatory bowel disease. Dig Liver Dis 2009; 41: 56-66.

42. Dhaliwal A, Zeino Z, Tomkins C, et al. Utility of faecal calprotectin in inflammatory bowel disease (IBD): what cut-offs should we apply? Frontline Gastroenterol 2015; 6: 14-19.

43. Herra OR, Christensen ML, Helms RA. Calprotectin: Clinical Applications in Pediatrics. J Pediatr Pharmacol Ther 2016; 21 : 308-321.

44. Ezri J, Nydegger A. Pediatrics. Fecal calprotectin in children: use and interpretation. Rev Med Suisse 2011; 7: 69-70.

45. Waligora-Dupriet AJ, Campeotto F, Romero K. Diversity of gut Bifidobacterium species is not altered between allergic and nonallergic French infants. Anaerobe 2011; 17: 91-96.

46. Daniluk U, Daniluk J, Krasnodebska M, et al. The combination of fecal calprotectin with ESR, CRP and albumin discriminates more accurately children with Crohn's disease. Adv Med Sci 2018; 64: 9-14.

47. Beşer ÖF, Sancak S, Erkan T, et al. Can Fecal Calprotectin Level Be Used as a Markers of Inflammation in the Diagnosis and Follow-Up of Cow's Milk Protein Allergy? Allergy Asthma Immunol Res 2014; 6: 33-38.

48. Kukkonen K, Kuitunen M, Haahtela T. High intestinal IgA associates with reduced risk of IgE-associated allergic diseases. Pediatr Allergy Immunol 2010; 21: 67-73.

49. Rosenberg HF. Eosinophil-derived Neurotoxin/RNase 2: connecting the past, the present and the future. Curr Pharm Biotechnol 2008; 9: 135-140.

50. Rosenberg HF, Domachowke JB. Eosinophils, eosinophil ribonucleases, and their role in host defence against respiratory virus pathogens. J Leukoc Biol 2001; 70: 691-698.

51. Kim CK, Callaway Z, Park JS, et al. Utility of serum eosinophilderived neurotoxin (EDN) measurement by ELISA in young children with asthma. Int Allerg 2017; 66: 70-74.

52. Majamaa H, Laine S, Miettinen A. Eosinophil protein X and eosinophil cationic protein as indicators of intestinal inflammation in infants with atopic eczema and food allergy. Clin Exp Allergy 1999; 29: 1502-1506.

53. Ada T, Toma T, Muraoka M. Elevation of fecal eosinophil-derived neurotoxin in infants with food protein-induced enterocolitis syndrome. Pediatr Allergy Immunol 2014; 25: 617-619.

54. Morita H, Nomura I, Shoda T. Fecal Eosinophil- derived neurotoxin is significantly elevated in non- IgE dependent Gastrointestinal allergies, especially in subtypes showing bloody stool [abstract]. J Allergy Clin Immunol 2012; 129: A353.

55. Aggarwal BB, Gupta SC, Kim JH. Historical perspectives on tumor necrosis factor and its superfamily: 25 years later, a golden journey. Blood 2012; 119: 651-665.

56. Heyman M, Darmon N, Dupont C, et al. Mononuclear cells from infants allergic to cow's milk secrete tumor necrosis factor alpha, altering intestinal function. Gastroenterology 1994; 106: 15141523.
57. Rodriguez P, Heyman M, Candalh C, et al. Tumour necrosis factor-alpha induces morphological and functional alterations of intestinal HT29 cl.19A cell monolayers. Cytokine 1995; 7: 441-448.

58. Chung HL, Hwang JB, Park JJ, et al. Expression of transforming growth factor beta1, transforming growth factor type I and II receptors, and TNF-alpha in the mucosa of the small intestine in infants with food protein-induced enterocolitis syndrome. J Allergy Clin Immunol 2002; 109: 150-154.

59. Wada H, Horisawa T, Inoue M, et al. Sequential measurement of fecal parameters in a case of non-immunoglobulin E-mediated milk allergy. Pediatr Int 2007; 49: 109-111.

60. Morita H, Nomura I, Orihara K, et al. Antigen-specific T-cell responses in patients with non-IgE-mediated gastrointestinal food allergy are predominantly skewed to $\mathrm{T}(\mathrm{H}) 2$. J Allergy Clin Immunol 2013; 131: 590-592. 